\title{
LETTERS
}

doi:10.1017/\$1041610209009132

\section{Changes in the perception of dementia in Japan}

In parallel with the aging of Japanese society, the number of older Japanese with dementia has been steadily increasing. In response to this trend, the Japanese government has established long-term care insurance and an adult guardianship system. At the same time, national and local governments, medical associations, the Alzheimer's Association and pharmaceutical companies have tried to promulgate knowledge regarding dementia. It is therefore important to assess whether these social movements are having an impact on the perception of dementia among the general population in Japan.

In 2004 we surveyed both younger and older citizens in some urban areas of Japan to determine their perceptions regarding dementia (Umegaki et al., 2007). The survey revealed that the perception of dementia was very similar between older and younger individuals, although the perceptions of the older individuals tended to be slightly more "inaccurate" and "negative." This result was in agreement with the report by Tanaka et al. (2004), which showed that age is a significant factor for stigma against mental disorders in Japan. In the current study, we resurveyed the same urban area after an interval of four years to determine the changes in the perception of dementia.

Two thousand younger residents aged 4064 years and 5,000 older residents aged 65 or older were randomly selected for receipt of a structured questionnaire by mail in December 2004, and an identically sized random selection and mailing were made in the same area in August 2008. People residing in long-term care facilities and those admitted to hospitals were excluded. The questionnaire included items about the backgrounds of participants and their perceptions regarding dementia. Both surveys were conducted in Nagoya City, which is located in the central part of Japan. In April 2004 the population of Nagoya City was $2,191,864$, with $17.7 \%$ of residents being aged 65 years or older; in 2008 , the population was $2,236,844$, with $19.9 \%$ being 65 or older.

A total of 942 younger residents $(47.1 \%)$ and $3273(65.5 \%)$ older residents returned the questionnaire in 2004 , and 950 younger $(47.5 \%)$ and 3132 older $(62.6 \%)$ residents answered the questionnaire in 2008 (Table 1). The backgrounds of the respondents were similar between the two surveys. Among the older residents who responded in 2004, 173 were excluded because they had been admitted to hospitals $(\mathrm{n}=53)$, were residing in long-term care facilities $(n=85)$, had moved or had died $(n=5)$, or because the address of residence was unknown $(n=9)$. In the 2008 survey, 145 were excluded because they had been admitted to hospital $(\mathrm{n}=39)$, were residing in long-term care facilities $(n=85)$, had moved or had died $(n=8)$.

The perceptions of dementia in 2004 and 2008 are shown in Table 2 . The four possible responses (yes, tend to yes, tend to no, no) were grouped into two categories (yes or tend to yes; no or tend to no), and $\chi^{2}$ analysis was performed. In younger respondents, the ratio between the two combinedresponse categories changed significantly for three of the nine items, whereas in older respondents, significant changes in the ratio between the two combined-response categories were found for seven of the nine items after four years. While the ratio of both older and younger respondents who answered that anyone may suffer from dementia (i.e. who answered yes or tend to yes on this item) increased, the ratio of those who answered that dementia is unrecognized by society and is untreatable significantly decreased. For three items (dementia is an unfamiliar and scary disease, dementia is a fatal disease, and dementia is a shameful disease), a significant decrease in answering yes or tend to yes was found only in the older responders.

The current survey revealed that the perception of dementia in an urban area in Japan has clearly changed over the past four years. The results may suggest that changes in the perception of dementia

Table 1. The gender and age characteristics of the two survey populations in 2004 and 2008 (values are $\mathrm{n}(\%))$

\begin{tabular}{|c|c|c|c|c|}
\hline & \multicolumn{2}{|c|}{ YOUNGER } & \multicolumn{2}{|c|}{ OLDER } \\
\hline & 2004 & 2008 & 2004 & 2008 \\
\hline \multicolumn{5}{|l|}{ Gender } \\
\hline Male & $395(42.3)$ & $388(40.8)$ & $1403(45.6)$ & $1260(42.2)$ \\
\hline Female & $538(57.7)$ & $561(59.2)$ & $1673(54.4)$ & $1723(57.8)$ \\
\hline \multicolumn{5}{|c|}{ Age (years) } \\
\hline $40-49$ & $306(32.5)$ & 339 (37.8) & & \\
\hline $50-59$ & $394(41.9)$ & $410(43.2)$ & & \\
\hline $60-64$ & $241(25.6)$ & $181(19.0)$ & & \\
\hline $65-69$ & & & $934(30.3)$ & $851(28.5)$ \\
\hline $70-79$ & & & $1548(50.3)$ & $1486(49.8)$ \\
\hline $80-89$ & & & $541(17.6)$ & $571(19.1)$ \\
\hline $90+$ & & & $57(1.9)$ & $79(2.6)$ \\
\hline
\end{tabular}


Table 2. Changes in the perception of dementia

\begin{tabular}{|c|c|c|c|c|c|c|}
\hline & \multirow[b]{2}{*}{ YEAR } & \multicolumn{3}{|c|}{ YOUNGER } & \multicolumn{2}{|c|}{ OLDER } \\
\hline & & $\begin{array}{l}\text { YES OR } \\
\text { TO YES }\end{array}$ & $\begin{array}{l}\text { TEND } \\
(\%)\end{array}$ & P VALUE & $\begin{array}{l}\text { YES OR TEND } \\
\text { TO YES }(\%)\end{array}$ & P VALUE \\
\hline \multirow[t]{2}{*}{ Morbid condition } & 2004 & 91.2 & & 0.128 & 86.8 & 0.149 \\
\hline & 2008 & 92.9 & & & 85.8 & \\
\hline \multirow[t]{2}{*}{ Disease of rising frequency } & 2004 & 92.2 & & 0.179 & 90.6 & $0.006^{* *}$ \\
\hline & 2008 & 93.5 & & & 88.3 & \\
\hline \multirow{2}{*}{ Anyone may suffer from dementia } & 2004 & 86 & & $<0.001^{* *}$ & 78.3 & $0.008^{* *}$ \\
\hline & 2008 & 93.1 & & & 81 & \\
\hline \multirow[t]{2}{*}{ Unfamiliar and scary disease } & 2004 & 76.9 & & 0.172 & 84 & $0.009^{* *}$ \\
\hline & 2008 & 74.9 & & & 81.4 & \\
\hline \multirow[t]{2}{*}{ Familiar disease } & 2004 & 81.7 & & 0.487 & 83.6 & 0.088 \\
\hline & 2008 & 81.9 & & & 82.2 & \\
\hline \multirow[t]{2}{*}{ Fatal disease } & 2004 & 69.8 & & 0.353 & 85.1 & $<0.001^{* *}$ \\
\hline & 2008 & 68.9 & & & 65.2 & \\
\hline \multirow[t]{2}{*}{ Disease unrecognized by society } & 2004 & 74.2 & & $<0.001^{* *}$ & 78.2 & $<0.001^{* *}$ \\
\hline & 2008 & 66.9 & & & 69.7 & \\
\hline \multirow[t]{2}{*}{ Shameful disease } & 2004 & 42.4 & & 0.179 & 57 & $0.004^{* *}$ \\
\hline & 2008 & 39.1 & & & 53.2 & \\
\hline \multirow[t]{2}{*}{ Untreatable disease } & 2004 & 29.1 & & $0.026^{*}$ & 39.2 & $<0.001^{* *}$ \\
\hline & 2008 & 24.9 & & & 31.9 & \\
\hline
\end{tabular}

$* \mathrm{p}<0.05$.

$* \mathrm{P}<0.01$

in the general population have been directed toward a more "accurate" or "positive" understanding of the disease. In particular, the reduction in the ratio of people who think that dementia is untreatable has some significance with regard to public health. As more people recognize that dementia is a treatable disease, patients with dementia will have a greater chance of receiving treatment. The changes in perception observed in the elderly participants appear to have been greater than those in the younger, with elderly participants showing three more items that changed significantly than the younger participants.

Why have perceptions changed over such a short period? During the interval between the two questionnaires, the aging of Japanese society accelerated, with the proportion of those aged 65 or older growing from $19.5 \%$ in 2004 to $22.0 \%$ in 2008 , and $17.7 \%$ to $19.9 \%$ in Nagoya City, where the current surveys took place. The rapid increase in the number of individuals with dementia is synchronous with the above demographic changes, and the dissemination of the public long-term care system must surely have raised public awareness (Fukuda et al., 2008). In addition, both national and local administrative efforts to spread knowledge regarding dementia may have contributed to the observed changes in perception (Schomerus et al., 2006). Another possible explanation for the current results is that a substantial number of older individuals who held unfavorable attitudes regarding dementia died in the interval between surveys. However, because this interval was only four years, this explanation seems unlikely.

Despite the favorable changes, the current study also showed that approximately $40 \%$ of younger and $50 \%$ of older individuals still think that dementia is a shameful condition. Although the trend is changing, it is clear that some prejudice against individuals with dementia is still present in the Japanese population, and these trends are similar to the results of recent surveys in Australia regarding mental disorders (Jorm et al., 2006).

The primary limitation of the current study was that it was carried out in only one urban area. Surveys in rural areas, where access to the media and community values are different, as well as surveys in other countries, where cultural and social backgrounds are different, would further illuminate the current results.

The current survey, which was carried out in an urban area of Japan, suggests that public perceptions of dementia may be changing rather more rapidly than expected, especially in the older population.

\section{Conflict of interest}

None. 


\section{Acknowledgments}

The authors thank the members of Nagoya City Hall for their cooperation.

\section{References}

Fukuda, Y., Nakao, H., Yahata, Y. and Imai, H. (2008). In-depth descriptive analysis of trends in prevalence of long-term care in Japan. Geriatric Gerontology International, $8,166-171$.

Jorm, A. F., Christensen, H. and Griffiths, K. M. (2006). The public's ability to recognize mental disorders and their beliefs about treatment: changes in Australia over 8 years. Australian and New Zealand Fournal of Psychiatry, 40, 36-41.

Schomerus, G., Matschinger, H., Kenzin, D., Breier, P. and Angermeyer, M. C. (2006). Public attitudes towards mental patients: a comparison between Novosibirsk, Bratislava and German cities. European Psychiatry, 21, 436-441.

Tanaka, G., Inadomi, H., Kikuchi, Y. and Ohta, Y. (2004) Evaluating stigma against mental disorder and related factors. Psychiatry Clinical Neuroscience, 58, 558566.

Umegaki, H., Onishi, J., Suzuki, Y., Endo, H. and Iguchi, A. (2007). Attitudes toward disclosing the diagnosis of dementia in Japan. International Psychogeriatrics, 19, 253-265.

HiROYUKI UMEGAKI, ${ }^{1}$ YUSUKE SUZUKI, JOJI OHNISHI $^{3}$ AND AKIHISA IGUCHI ${ }^{4}$

${ }^{1}$ Department of Geriatrics, Nagoya University Graduate School of Medicine, 65 Tsuruma-cho, Showa-Ku, Nagoya, Aichi, 466-8550, Japan Email: umegaki@med.nagoya-u.ac.jp

${ }^{2}$ Department of Home Care Management, Nagoya University Hospital, 65 Tsuruma-cho, Showa-Ku, Nagoya, Aichi, 466-8550, Japan

${ }^{3}$ Department of Gero-informatics, Nagoya University School of Medicine, Daiko Medical center, Daiko minami 1-1-20, Higasi-ku Nagoya, 461-0047, Aichi, Japan

${ }^{4}$ Department of Community Care Philanthropy, Faculty of Medical Welfare Sakuragaoka 23, Chikusa-ku, Nagoya, 464-8671, Aichi, Japan

the 32 patients $(75 \%)$ and atrophy of hippocampus was seen in $15(46.8 \%)$ out of the 32 patients. In all except one, atrophy was associated with hypertension. Family history and history of head injury was present in two cases each (12.5\%), and nine cases were diabetic $(28.1 \%)$. Six patients $(18.75 \%)$ had a history of coronary artery disease, and $15(46.8 \%)$ had behavioral and psychological symptoms of dementia. Twenty patients were graduates, six $(18.75 \%)$ illiterate and another six $(18.75 \%)$ were postgraduates. There was no history of trauma or epileptic fits in any of them.

Hipppcampal atrophy is an important milestone in $\mathrm{AD}$. Studies indicate that atrophy of this region correlates well with cognitive decline in $\mathrm{AD}$ patients (den Heijer et al., 2005). We have previously reported hippocampal atrophy as a seizure predictor (Dhikav and Anand, 2007), and a large study of over 500 subjects with no dementia showed that blood pressure and indicators of small-vessel disease in the brain may be associated with the atrophy of structures affected by AD pathology (Yavuz et al., 2006).

The study by Wiseman et al. (2004) involving 103 hypertensive patients showed an adverse influence of hypertension on brain structures. Although patients in the present study had more factors that could potentially contribute to hippocampal atrophy, hypertension is the main one. The mechanism by which hypertension could be contributing towards atrophy of hippocampus is unknown, but we assume that hypo-perfusion and hypoxia of the hippocampus may be involved. If 